CIENCIAMATRIA

Revista Interdisciplinaria de Humanidades, Educación, Ciencia y Tecnología

Año V. Vol. V. N9. Julio - Diciembre 2019

Hecho el depósito de ley: pp201602FA4721

ISSN-L: 2542-3029; ISSN: 2610-802X

Universidad Nacional Experimental Francisco de Miranda (UNEFM). Santa Ana de Coro. Venezuela

Carlos Enrique Santis Loor; Alba Verónica Barreto Pincay; Flor Maria Bailón López; Maria Monserrate Bravo Guerrero

DOI $10.35381 / \mathrm{cm} . v 5 i 9.250$

\title{
Clima organizacional en ambientes universitarios
}

\section{Organizational climate in university environments}

\author{
Karina Elizabth Mera Vinces \\ kemv2121@gmail.com \\ Instituto Tecnológico Superior Luis Arboleda Martínez, Manta \\ Ecuador \\ https://orcid.org/0000-0003-2139-1090 \\ Yesenia Alexandra Mendoza Espinoza \\ yesy9379@gmail.com \\ Instituto Tecnológico Superior Luis Arboleda Martínez, Manta \\ Ecuador \\ https://orcid.org/0000-0002-2125-4638 \\ Carolina Elizabeth Pinto Bayas \\ caropinto14@hotmail.com \\ Instituto Tecnológico Superior Luis Arboleda Martínez, Manta \\ Ecuador \\ https://orcid.org/0000-0003-0106-624X
}

Recibido: 13 de agosto de 2019

Aprobado: 15 de septiembre de 2019

\begin{abstract}
RESUMEN
La investigación se basó en descripción de los hechos tal como ocurren en la realidad, con la finalidad de analizarlos producto de la aplicación de la estadística descriptiva, para lo cual se empleó el tipo descriptiva de campo no experimental con el objetivo de analizar el clima organizacional en ambientes universitarios de la ciudad de Manta - Ecuador. El clima organizacional que tiene mayor énfasis está relacionado al autoritario, lo que implica, que los jefes son los que dirigen de un modo unidireccional, es decir, no aceptan sugerencias, ni son consultivos, sobre las decisiones que se han de tomar en la organización.
\end{abstract}

Descriptores: Universidad; Trabajador; Organización; Empresa sin ánimo de lucro. 


\begin{abstract}
The investigation was based on a description of the facts as they occur in reality, in order to analyze them as a result of the application of descriptive statistics, for which the descriptive type of non-experimental field was used with the aim of analyzing the organizational climate in university environments of the city of Manta - Ecuador. The organizational climate that has the greatest emphasis is related to the authoritarian, which implies that the bosses are the ones who lead in a unidirectional way, that is, they do not accept suggestions, nor are they consultative, about the decisions to be taken in the organization.
\end{abstract}

Descriptors: Universities; Workers; Organizations; Nonprofit organizations.

\title{
INTRODUCCIÓN
}

El ambiente laboral es una sumatoria de factores externos e internos que hacen posible la existencia de un espacio para el trabajo, pero este, no puede ser simplemente un espacio, sino, que debe constituirse realmente en un ambiente que permita trabajar del mejor modo posible, en este sentido, Iglesias (2008) plantea que "el ambiente es el conjunto del espacio físico y las relaciones que se establecen en él" (p. 254).

Por su parte, Ramírez (2005) manifiesta que "el ambiente de trabajo es el factor esencial en el rendimiento humano. Éste tiende a determinarse a medida que transcurre el tiempo, unas veces como consecuencia de la fatiga y otras como resultado del aburrimiento y la falta de motivación" (p. 195). Partiendo de lo expuesto, se tiene que el ambiente de trabajo debe ser reestructurado en ciertas ocasiones con el fin de no causar agotamiento en el empleado, por lo tanto, la comunicación eficaz en este sentido puede contribuir a que tanto empleados como empleadores puedan aportar en la construcción de un ambiente de trabajo acorde a las exigencias de todos quienes laboran en la empresa.

Esta situación, podría fomentar la productividad de la empresa, por el simple hecho de que los empleados se sienten tomados en cuenta para la construcción del ambiente de trabajo para el cual laboran, en este punto, podría entenderse incluso como una medida de reconocimiento por parte de los empleadores. En este sentido, Cavassa (2005) al igual 
que Ramírez, plantea que el ambiente de trabajo es un factor esencial para el rendimiento laboral; por lo que es necesario que el hombre no trabaje más allá de los límites de su resistencia y en condiciones ambientales adecuadas.

De acuerdo a lo planteado, debe entender que no solo el aspecto físico y ergonómico de la empresa comprenden el ambiente de trabajo, sino, que la motivación que tenga el empleado también se encuentra dentro del ambiente de trabajo, en este sentido, Brunet (2004) señala que el ambiente laboral "es un componente multidimensional de elementos al igual que el clima atmosférico" (p. 12). Por lo tanto, los gerentes deben cuidar una serie de factores que van desde el aspecto físico de la empresa hasta la comunicación que se utiliza en la misma, por cuanto, Gotera (2005) manifiesta que el ambiente "tiende a mostrarse favorable y positivo si no existen problemas; si el clima organizacional frustra la satisfacción de las necesidades de los miembros; el ambiente organizacional tiende a mostrarse desfavorable y negativo" (p. 25).

Partiendo de lo expuesto, se convierte en un reto para la gerencia de la empresa emprender acciones que conlleven a mantener un ambiente de trabajo favorable, por cuanto así, evitarán que se creen conflictos en el seno de la misma, por el contrario al tener presente la acentuación de un ambiente de trabajo óptimo, podrán verse favorecidos en la productividad y en la calidad de los servicios que prestan.

Lo expuesto no escapa a las instituciones universitarias, por cuanto en ella labora un talento humano que necesita laboral en un clima organizacional óptimo con la finalidad de promover un trabajo eficaz en función de cumplir con las metas propuestas por la organización, siendo indispensable como se genera en las instituciones educativas universitarias de la ciudad de Manta - Ecuador, el clima organizacional, lo cual permitirá focalizar aspectos relevantes y debilidades con la finalidad de proseguir profundizando en investigaciones sub siguientes que permitan aplicar medidas en pro de transcender a un ambienta laboral favorable para el abordaje de los objetivos planteados.

En prosecución con lo planteado, se formula el objetivo de analizar el clima organizacional en ambientes universitarios de la ciudad de Manta - Ecuador. 


\section{DESARROLLO}

\section{Clima organizacional}

El clima organizacional es un factor primordial en el desarrollo de la empresa, cuando esta posee un óptimo clima organizacional tiende a transitar hacia el éxito, puede señalarse que el trabajo individual y grupal se eleva en beneficio de la organización, en este sentido, Robbins (2004) señala que el clima organizacional "es un ambiente compuesto de las instituciones y fuerzas externas que pueden influir en su desempeño" (p. 87), es decir que el ambiente pudiera afectar la estructura de las organizaciones.

Esta estructura viene representada por la productividad que puedan desarrollar los agentes que hacen vida en la empresa, la cual es influenciada desde la comunicación eficaz que pueda implementarse en la organización, por lo tanto, el clima organizacional es un ambiente que se crea más allá del aspecto físico que condiciona a la empresa, el verdadero clima organizacional es que es promovido desde los empleados y por los empleados, se puede decir, que es un ambiente que se estructura bajo las perspectivas que posean las personas que conforman una empresa.

En este sentido, Brunet (2007) señala que el clima organizacional "constituye una configuración de las características de una organización, así como las características personales de un individuo pueden constituir su personalidad" (p. 12). Partiendo de lo expuesto, se tiene que el clima organizacional tiene que ver directamente con la personalidad de los empleados o que esta tiene que ver con el clima organizacional, es decir, ambas se configuran para producir el clima organizacional.

Desde esta perspectiva, se hace importante que en la empresa se promueva la motivación por medio de la comunicación de todas las personas que hacen vida en la empresa, de ese modo, podrá mayor posibilidad de que las personalidades trabajen en una meta en común, que no es otra cosa, que las metas propuestas por la empresa. Goncalves (2002) describe que es necesario que la gerencia de la empresa establezca mecanismos que propicien un óptimo clima organizacional, pero más aún, que estos 
Universidad Nacional Experimental Francisco de Miranda (UNEFM). Santa Ana de Coro. Venezuela

Carlos Enrique Santis Loor; Alba Verónica Barreto Pincay; Flor Maria Bailón López; Maria Monserrate Bravo Guerrero

medios incentiven el conocimiento de los factores que intervienen en el clima organizacional, en este sentido, plantea:

El conocimiento del clima organizacional proporciona retroinformación acerca de los procesos que determinan los comportamientos organizacionales, permitiendo además, introducir cambios planificados tanto en las actitudes y conductas de los miembros, como en la estructura organizacional o en uno o más de los subsistemas que la componen (p. 47).

En este sentido, se tiene que el clima organizacional puede promover actitudes de compromiso de los empleados con las metas que se proponen en la organización, por lo tanto, la gerencia debe generar mecanismos que le conlleven a propiciar un óptimo clima organizacional donde los empleados puedan percibir y confianza y así, iniciar un proceso de retroinformación que exprese las fortalezas y debilidades de la organización, de ese modo, podrá tomarse los correctivos necesarios para mejorar la productividad.

En este punto, la gerencia puede promover una comunicación eficaz con el fin de incentivar un óptimo clima organizacional, por lo tanto, la comunicación y el clima organizacional, podrían generar un efecto sinérgico que beneficiaría a la organización en cuanto a la calidad y productividad de la misma. Es así, que generar una percepción agradable en los empleados del clima organizacional que se vive en la empresa es de suma importancia para encaminar a la consecución de metas propuestas desde la gerencia para la consolidación operativa de la organización.

En este sentido, Likert citado por Brunet (2007) sostiene que en la percepción del clima de una organización influyen variables tales como la estructura de la organización y su administración; las reglas y normas; la toma de decisiones, entre otros elementos. Estas son variables causales las cuales determinan el sentido de como una organización evoluciona así como los resultados que obtiene.

Otro de los elementos que se debe tener en cuenta en la influencia del clima organizacional, como se ha venido comentando, es la comunicación eficaz, por cuanto en mejor proporción esta se pueda desarrollar en la empresa, en mejor calidad puede darse la relación entre empleados y entre éstos con sus jefes; por el contrario, no tener 
una comunicación acorde, podría traer inconvenientes en la convivencia laboral y verse así, afectado el clima organizacional de la empresa con lo cual la productividad de la misma, podría verse afectada negativamente.

Partiendo de lo expuesto, Sandoval (2004) define el clima organizacional como la "cualidad o propiedad de ambiente organizacional que es percibido o experimentado por los miembros de la organización y que influyen en su comportamiento" (p. 210). Por lo tanto, la experiencia que perciban los empleados en la empresa, debe ser la más óptima con el fin de condicionar un ambiente favorable para el trabajo con lo cual, existirá mayor probabilidad de que la empresa camine hacia el éxito, la calidad y productividad.

Por otro lado, Goncalves (2000) considera que, de todos "los enfoques sobre el concepto de clima organizacional, el que ha demostrado mayor utilidad es el que utiliza como elemento fundamental las percepciones que el trabajador tiene de las estructuras y procesos que ocurren en un medio laboral" (p. 79). En este sentido, el clima organizacional no solo debe entenderse como el ambiente de trabajo que engloba a la empresa, sino, que debe verse como el ambiente interno que se genera el empleado en sí mismo y del ambiente que le rodea, es decir, se trata de un tema de percepciones que debe ser visto y entendido desde la visión del empleado.

Debido que es éste quien debe realizar el trabajo fuerte en la empresa, por lo tanto, es menester que se sienta a gusto con la misma y con sus compañeros de trabajo, es ahí, donde la gerencia debe impulsar planes de crecimiento que optimicen la percepción de los empleados sobre el clima organizacional, puesto que esta postura le garantizará un mayor éxito en las operaciones que la empresa emprende día a día.

\section{Tipos de clima organizacional}

El clima organizacional es generado desde la gerencia y esta acción es percibida por los empleados, de cómo éstos la perciban, va a depender en gran medida sí la calidad y productividad se eleva o por el contrario puede disminuir, también esta percepción tiene que ver con las motivaciones internas que pueda tener el empleado a la hora de realizar 
un determinado trabajo, en consideración el clima organizacional juega un papel importante en el desarrollo de la organización.

Por otro lado, los empleados en algún momento pueden generar su propio clima organizacional, la diferencia radica es que este puede estar muchas veces en contrariedad con los planes de la gerencia, lo cual conlleva a una ruptura virtual de las relaciones entre ambos, perjudicándose así, la calidad y productividad de la organización, en este sentido, la comunicación que se ejerza para considerar todos los ambientes que se desarrollan en la empresa, jugará un papel importante en la optimización de la misma, es así, que se hace importante estudiar los diversos climas organizacionales que puedan desarrollarse en la empresa:

\section{Autoritario Explotador}

Desde lo expuesto por Likert citado por Brunet (2007), este tipo de sistema se caracteriza por la desconfianza, debido a que "la organización no tiene confianza hacia sus empleados" (p. 30). Las decisiones son adoptadas en la cumbre de la organización y desde allí se difunden siguiendo una línea altamente burocratizada de conducción regular, donde los empleados tienen miedo, no son plenamente reconocidos y sienten que su trabajo no es recompensado. Los procesos de control se encuentran también centralizados y formalizados, la comunicación solo se da entre los superiores y se distribuye según una función puramente descendiente.

En este tipo, de clima no se estudia la percepción que el empleado tiene sobre la empresa, las decisiones vienen dadas de un modo unilateral y simplemente deben ser cumplidas, la línea de comunicación puede ser escrita y la gerencia muchas veces no se involucrada directamente con los empleados, por lo tanto, no puede darse una comunicación empática que invite al logro por parte de los empleados a realizar la actividad encomendada.

Esto puede traer como consecuencia que sean los mismos empleados, quienes generen clima organizacional, el cual puede ser óptimo o no para la empresa, ante esta situación, la gerencia no puede tener un control efectivo sobre el ambiente de trabajo, lo cual puede 
generar situaciones que no sean las más beneficiosas para la empresa. En este sentido, Robbins (2004) expone que este tipo de clima organizacional es gerenciado por un liderazgo autocrático el cual es definido desde dos visiones:

Autocrático I (Al): usted mismo soluciona el problema o toma una decisión usando cualesquiera hechos que tenga a la mano Autocrático II (All): usted obtiene la información necesaria de los subordinados y entonces decide la solución del problema. Podría o no decirles acerca de la naturaleza de la situación que enfrenta. Usted busca de ellos únicamente los hechos relevantes no su opinión o consejo (p. 273).

Como puede apreciarse, en este tipo de clima organizacional es la gerencia quien únicamente toma la decisión a seguir, no se toma en cuenta la postura de los empleados, quienes en un momento determinado por ser quienes conocen una realidad en particular de la empresa, pudiesen brindar mayor información que permitiese elaborar planes que conlleven a una mejor operatividad de la empresa, por consiguiente, utilizar este tipo de clima de un modo constante en la empresa, puede traer consigo que los empleados tiendan a la desmotivación por cuanto su percepción nunca es tomada en cuenta para el crecimiento de la organización.

\section{Paternalista}

En este clima organizacional las decisiones son generadas por la gerencia de la organización; sin embargo, esta tiene confianza en su personal, también en este sistema se centraliza el control, pero en él hay una mayor delegación que en el tipo anterior. E tipo de relaciones característico es paternalista, con autoridades que tienen todo el poder, pero concede ciertas facilidades a sus subordinados, fundamentadas dentro de límites de relativa flexibilidad.

Asimismo, en este tipo de clima según Likert citado por Brunet (2007), se puede evidenciar que se dan las recompensas y algunas veces los castigos, esto trae consigo que exista un mayor acercamiento entre los jefes y los empleados; bien sea para recompensar o castigar una acción, sin embargo, en cualquiera de las situaciones, la utilización de la comunicación eficaz se hace pertinente con el fin de que el mensaje sea 
el más claro, objetivo, empático, y así, pueda verse y entenderse como verdaderamente una invitación a una mejor producción de la empresa.

\section{Consultivo}

Este tipo de clima organizacional se caracteriza por tener una mayor delegación de las decisiones, puesto que la política y las decisiones aunque se toman generalmente en la cima permiten la opinión de los subordinados. Likert citado por Brunet (2007) refiere que se mantiene un esquema jerárquico, pero las decisiones específicas son adoptadas por escalones medios e inferiores. También el control es delegado a escalones inferiores. Robbins (2005), plantea esta visión como un clima organizacional gerenciado por un liderazgo consultivo, definido desde dos visiones:

Consultivo I (Cl): usted comparte en forma individual el problema con los subordinados relevantes, obtiene sus ideas y sugerencias. Sin embargo, la decisión final es suya solamente. Consultivo II (CII): usted comparte el problema con sus subordinados como un grupo, obteniendo colectivamente sus ideas y sugerencias. Entonces usted toma la decisión que podría o no reflejar la influencia de sus subordinados (p. 274).

Este tipo de clima toma las decisiones en los puntos intermedios de la empresa, esto hace que pueda existir una mayor clasificación de la información que se tiene sobre un determinado evento en la organización y que los empleados puedan expresar su opinión. Esto conlleva a tener un clima organizacional donde el empleado se siente tomado en cuenta, con lo cual su motivación puede verse influenciada positivamente, sin embargo, la comunicación eficaz, debe ser vista como un elemento para la consolidación de los planes y metas que se propongan en la organización.

\section{Participativo}

Partiendo de lo expuesto por Likert citado por Brunet (2007), este tipo de clima organizacional se caracteriza porque "el proceso de toma de decisiones no se encuentra centralizado, sino distribuido en diferentes lugares de la organización, donde la dirección tiene plena confianza en sus empleados" (p. 31). Las comunicaciones son tanto verticales 
como horizontales, generándose una partida grupal. El clima de este tipo de organización logra altos niveles de compromiso con los trabajadores, la organización y sus objetivos. La comunicación no se hace solamente de manera ascendente o descendente, sino también de forma lateral.

En este sentido, para Robbins (2004), este tipo de liderazgo fomenta "la participación del equipo en la toma de decisiones" (p. 275). Sin embargo, para que se pueda desarrollar este tipo de clima organizacional de un modo efectivo, se hace necesario que los miembros que conforman la organización, posean una cuota de madurez que se traduce en responsabilidad, pro actividad, empatía, comunicación eficaz, entre otros elementos, para que no exista distorsión entre lo que quiere una persona para el colectivo; este tipo de clima no plantea que cada quien pueda hacer lo que mejor le parezca, sino que debe cumplir principios y normas pero sin la rigidez de otros modelos.

Partiendo de lo expuesto, se tiene que este clima organizacional procura la participación de todos los agentes que hacen vida en la empresa, no se trata pues de permitir que cada empleado haga lo que mejor le parezca, sino, que cada quien pueda aportar lo mejor de sí para el crecimiento de la empresa, en este punto la comunicación eficaz juega un papel fundamental para convertirse en un medio que propicie la integración de la gerencia y los empleados en pro de consolidar la calidad y productividad en la empresa.

\section{MÉTODO}

La investigación se basó en descripción de los hechos tal como ocurren en la realidad, con la finalidad de analizarlos producto de la aplicación de la estadística descriptiva, para lo cual se empleó el tipo descriptiva de campo no experimental (Hernández, Fernández y Baptista, 2014), aplicándose la encuesta como técnica de recopilación de datos y un cuestionario de 31 ítems tipo escalamiento de Likert, con la finalidad de conocer la percepción de 35 personas que forman parte del talento humano de la Universidad Laica "Eloy Alfaro" de Manabí (ULEAM), de ese modo, se correspondió la metodología con el objetivo general de la investigación analizar el clima organizacional en ambientes 


\section{CIENCIAMATRIA}

Revista Interdisciplinaria de Humanidades, Educación, Ciencia y Tecnología

Año V. Vol. V. N9. Julio - Diciembre 2019

Hecho el depósito de ley: pp201602FA4721

ISSN-L: 2542-3029; ISSN: 2610-802X

Universidad Nacional Experimental Francisco de Miranda (UNEFM). Santa Ana de Coro. Venezuela

Carlos Enrique Santis Loor; Alba Verónica Barreto Pincay; Flor Maria Bailón López; Maria Monserrate Bravo Guerrero

universitarios de la ciudad de Manta - Ecuador.

\section{DISCUSIÓN DE LOS RESULTADOS}

Clima tipo Autoritario - explotador.

Cuadro $\mathrm{N}^{\circ}-1$.

\begin{tabular}{|c|c|c|c|c|c|c|c|c|c|}
\hline \multicolumn{2}{|c|}{ SIEMPRE } & \multicolumn{2}{|c|}{$\begin{array}{c}\text { CASI } \\
\text { SIEMPRE }\end{array}$} & \multicolumn{2}{|c|}{$\begin{array}{l}\text { ALGUNAS } \\
\text { VECES }\end{array}$} & \multicolumn{2}{|c|}{$\begin{array}{c}\text { CASI } \\
\text { NUNCA }\end{array}$} & \multicolumn{2}{|c|}{ NUNCA } \\
\hline FA & $\%$ & FA & $\%$ & FA & $\%$ & FA & $\%$ & FA & $\%$ \\
\hline 8 & 23 & 5 & 14 & 3 & 9 & 14 & 40 & 5 & 14 \\
\hline
\end{tabular}

La opción siempre tuvo una representación estadística del 23\%, así mismo la opción casi siempre un $14 \%$, la alternativa algunas veces $9 \%$ y las opciones casi nunca $40 \%$ y nunca el $14 \%$

Puede señalarse que la mayoría de los encuestados expresan que no tienen confianza de expresarle sus ideas al jefe; así mismo, consideran que el jefe es quien decide lo que se hace en el trabajo. En este sentido; Likert citado por Brunet (2007), este tipo de sistema se caracteriza por la desconfianza, debido a que "la organización no tiene confianza hacia sus empleados" (p. 30).

Se hace necesario que en la organización se promueva un clima de confianza entre el talento humano que labora, por cuanto esto permite tener mayor capacidad a la hora de realizar los trabajos, debido que se cuenta con el apoyo necesario para el trabajo en 
equipo, así como también se promueve la cooperación y participación activa en promover una mayor y mejor productividad laboral.

Tipo de clima paternalista.

Cuadro $N^{\circ}-2$.

\begin{tabular}{|c|c|c|c|c|c|c|c|c|c|}
\hline \multicolumn{2}{|c|}{ SIEMPRE } & \multicolumn{2}{|c|}{$\begin{array}{c}\text { CASI } \\
\text { SIEMPRE }\end{array}$} & \multicolumn{2}{|c|}{$\begin{array}{l}\text { ALGUNAS } \\
\text { VECES }\end{array}$} & \multicolumn{2}{|c|}{$\begin{array}{c}\text { CASI } \\
\text { NUNCA }\end{array}$} & \multicolumn{2}{|c|}{ NUNCA } \\
\hline $\begin{array}{l}\text { FA } \\
4\end{array}$ & $\begin{array}{l}\% \\
11\end{array}$ & $\begin{array}{l}\text { FA } \\
3\end{array}$ & $\begin{array}{l}\% \\
9\end{array}$ & $\begin{array}{c}\text { FA } \\
5\end{array}$ & $\begin{array}{l}\% \\
14\end{array}$ & $\begin{array}{l}\text { FA } \\
10\end{array}$ & $\begin{array}{l}\% \\
29\end{array}$ & $\begin{array}{l}\text { FA } \\
13\end{array}$ & $\begin{array}{l}\% \\
37\end{array}$ \\
\hline
\end{tabular}

La opción siempre tuvo una representación estadística del 11\%, así mismo la opción casi siempre un $9 \%$, la alternativa algunas veces $14 \%$, casi nunca $29 \%$ y nunca con el $37 \%$ La gran mayoría de los encuestados, manifiestan que su jefe no le motiva cuando realiza un trabajo; así como también consideran que sus jefes en ocasiones no le supervisan al realizar tu trabajo. En este sentido; Likert citado por Brunet (2007), se puede evidenciar que se dan las recompensas y algunas veces los castigos, esto trae consigo que exista un mayor acercamiento entre los jefes y los empleados; bien sea para recompensar o castigar una acción.

Se evidencia la necesidad en la organización de promover recompensas y castigos en el talento humano con el fin de promover una aptitud competitividad por medio del trabajo cooperativo y co responsable, esto podría promover una mejor y mayor productividad en la organización.

Tipo de clima consultivo.

Cuadro $\mathrm{N}^{\circ}-3$.

\begin{tabular}{|c|c|c|c|c|c|c|c|c|c|}
\hline \multicolumn{2}{|c|}{ SIEMPRE } & \multicolumn{2}{|c|}{$\begin{array}{c}\text { CASI } \\
\text { SIEMPRE }\end{array}$} & \multicolumn{2}{|c|}{$\begin{array}{c}\text { ALGUNAS } \\
\text { VECES }\end{array}$} & \multicolumn{2}{|c|}{$\begin{array}{c}\text { CASI } \\
\text { NUNCA }\end{array}$} & \multicolumn{2}{|c|}{ NUNCA } \\
\hline FA & $\%$ & FA & $\%$ & FA & $\%$ & FA & $\%$ & $\overline{F A}$ & $\%$ \\
\hline 3 & 8 & 4 & 11 & 2 & 6 & 16 & 46 & 10 & 29 \\
\hline
\end{tabular}

La opción siempre tuvo una representación estadística del 8\%, así mismo la opción casi siempre un $11 \%$, la alternativa algunas veces $6 \%$, casi nunca $46 \%$ y nunca con el $29 \%$ 
Universidad Nacional Experimental Francisco de Miranda (UNEFM). Santa Ana de Coro. Venezuela

Carlos Enrique Santis Loor; Alba Verónica Barreto Pincay; Flor Maria Bailón López; Maria Monserrate Bravo Guerrero

La gran mayoría de los encuestados, consideran que su jefe no escucha sugerencias para mejorar el trabajo; así como tampoco, realiza reuniones para escuchar opiniones del personal sobre cómo mejorar el ambiente de trabajo. En este sentido; Robbins (2005: 274), plantea esta visión como un clima organizacional gerenciado por un clima consultivo, definido desde dos visiones:

Consultivo I (Cl): usted comparte en forma individual el problema con los subordinados relevantes, obtiene sus ideas y sugerencias. Sin embargo, la decisión final es suya solamente. Consultivo II (CII): usted comparte el problema con sus subordinados como un grupo, obteniendo colectivamente sus ideas y sugerencias. Entonces usted toma la decisión que podría o no reflejar la influencia de sus subordinados.

Surge la necesidad de fomentar una participación basada en el liderazgo consultivo, por cuanto este permite tener información directa de los empleados sobre los eventos que ocurren en la organización, así mismo, permite que los empleados expresen sus ideas de cómo mejorar la organización, siendo una oportunidad para que se sientan tomados en cuenta.

\section{Reconocimiento. \\ Cuadro $N^{\circ}-4$.}

\begin{tabular}{|c|c|c|c|c|c|c|c|c|c|}
\hline \multicolumn{2}{|c|}{ SIEMPRE } & \multicolumn{2}{|c|}{$\begin{array}{c}\text { CASI } \\
\text { SIEMPRE }\end{array}$} & \multicolumn{2}{|c|}{$\begin{array}{l}\text { ALGUNAS } \\
\text { VECES }\end{array}$} & \multicolumn{2}{|c|}{$\begin{array}{c}\text { CASI } \\
\text { NUNCA }\end{array}$} & \multicolumn{2}{|c|}{ NUNCA } \\
\hline FA & $\%$ & FA & $\%$ & FA & $\%$ & FA & $\%$ & FA & $\%$ \\
\hline 2 & 6 & 3 & 9 & 1 & 3 & 17 & 48 & 12 & 34 \\
\hline
\end{tabular}

La opción siempre tuvo una representación estadística del 6\%, así mismo la opción casi siempre un $9 \%$, la alternativa algunas veces $3 \%$, casi nunca $48 \%$ y nunca con el $34 \%$ La mayoría de las personas encuestadas, manifiestan que su jefe no le felicita cuando realizan un determinado trabajo; así como tampoco, sus compañeros de trabajo le comunican que ha logrado realizar un buen trabajo. Por lo tanto, no se evidencia que se genere un clima de reconocimiento en la organización. En este sentido; Robbins (2004) "consiste en la atención personal, mostrar interés, aprobación y aprecio por un trabajo 
CIENCIAMATRIA

Revista Interdisciplinaria de Humanidades, Educación, Ciencia y Tecnología

Año V. Vol. V. No9. Julio - Diciembre 2019

Hecho el depósito de ley: pp201602FA4721

ISSN-L: 2542-3029; ISSN: 2610-802X

Universidad Nacional Experimental Francisco de Miranda (UNEFM). Santa Ana de Coro. Venezuela

Carlos Enrique Santis Loor; Alba Verónica Barreto Pincay; Flor Maria Bailón López; Maria Monserrate Bravo Guerrero

bien hecho" (p. 279), partiendo de esta postura no puede apreciarse la el reconocimiento solo desde el punto de vista económico, sino, que debe tenerse en cuenta una palabra, un gesto, que impulse al empleado a seguir adelante, con confianza, en la labor que realiza, de ese modo, no solo crecerá individualmente, sino, la empresa también lo hará.

\section{Ambiente laboral.}

Cuadro $N^{\circ}-5$.

\begin{tabular}{|c|c|c|c|c|c|c|c|c|c|}
\hline \multicolumn{2}{|c|}{ SIEMPRE } & \multicolumn{2}{|c|}{$\begin{array}{c}\text { CASI } \\
\text { SIEMPRE }\end{array}$} & \multicolumn{2}{|c|}{$\begin{array}{l}\text { ALGUNAS } \\
\text { VECES }\end{array}$} & \multicolumn{2}{|c|}{$\begin{array}{c}\text { CASI } \\
\text { NUNCA }\end{array}$} & \multicolumn{2}{|c|}{ NUNCA } \\
\hline FA & $\%$ & FA & $\%$ & FA & $\%$ & FA & $\%$ & FA & $\%$ \\
\hline 6 & 17 & 7 & 20 & 4 & 12 & 13 & 37 & 5 & 14 \\
\hline
\end{tabular}

La opción siempre tuvo una representación estadística del 17\%, así mismo la opción casi siempre un $20 \%$, la alternativa algunas veces $12 \%$, casi nunca $37 \%$ y nunca con el $14 \%$ La mayoría considera que el ambiente de trabajo no es bueno para realizar tus actividades, aunque manifiestan que le brindan todas las condiciones para que realices tu trabajo. Esto implica que no se está generando un buen ambiente laboral, lo que implica la necesidad de gestionar un mejor clima organizacional. En este sentido; Ramírez (2005) manifiesta que "el ambiente de trabajo es el factor esencial en el rendimiento humano.

Por lo tanto; el ambiente de trabajo debe ser reestructurado en ciertas ocasiones con el fin de no causar agotamiento en el empleado, por lo tanto, la comunicación eficaz en este sentido puede contribuir a que tanto empleados como empleadores puedan aportar en la construcción de un ambiente de trabajo acorde a las exigencias de todos quienes laboran en la empresa. 


\section{CONCLUSIONES}

El clima organizacional que tiene mayor énfasis está relacionado al autoritario, lo que implica, que los jefes son los que dirigen de un modo unidireccional, es decir, no aceptan sugerencias, ni son consultivos, sobre las decisiones que se han de tomar en la organización.

Esto puede traer como consecuencia que el talento humano no se siente tomado en cuenta para aportar ideas que contribuyan a fortalecer la productividad que se desarrolla en la organización, así mismo, puede traer desmotivación entre el talento humano quien puede percibir que sus talentos y competencias no son puestas al servicio, con lo cual, los aportes que pueden realizar se circunscriben a realizar las tareas propias de su cargo y a cumplir con las ordenes que dictan los jefes en función de lo que se considera oportuno para el crecimiento de la organización, siendo necesario visualizar la satisfacción del cliente desde la prestación de un servicio de calidad, Aldana\& Piña (2017).

Para mejorar el clima organizacional, se debe tener en cuenta la incorporación de formación al talento humano en temas relacionados a mejorar el ambiente laboral, por lo tanto, no solo se deben abordar tópicos sobre clima organizacional, sino, de convivencia y crecimiento personal con el fin de promover acciones que contribuyan a fundamentar y establecer un óptimo ambiente laboral en la organización.

\section{REFERENCIAS CONSULTADAS}

1. Aldana, J., \& Piña, J. (2017). Calidad del servicio prestado al cliente por los instructores de gimnasios. Revista Arbitrada Interdisciplinaria Koinonía, 2(3), 172197. http://fundacionkoinonia.com.ve/ojs/index.php/revistakoinonia/article/view/59/46

2. Brunet, L. (2007). El Clima en las Organizaciones. Editorial Panapo. México.

3. Cavassa, C. (2005). Seguridad Industrial un Enfoque Integral. México: Limusa. 
Universidad Nacional Experimental Francisco de Miranda (UNEFM). Santa Ana de Coro. Venezuela

Carlos Enrique Santis Loor; Alba Verónica Barreto Pincay; Flor Maria Bailón López; Maria Monserrate Bravo Guerrero

4. Gotera, F. (2005). Comunicación en las organizaciones. Editorial Panapus. Buenos Aires. Argentina.

5. Goncalves (2002). Fundamentos del Clima Organizacional. Sociedad Latinoamericana para la Calidad. (SLC).

6. Hernández, Fernández y Baptista (2014). Metodología de la investigación. México, Mc Graw Hill Hispanoamericana. Hill Internacional

7. Iglesias, F. (2008), Observación y Evaluación del Ambiente de Aprendizaje en Educación Infantil: Dimensiones y Variables a Considerar. Revista Iberoamericana de Educación. Publicación editada por la OEI № 47

8. Ramírez, F. (2005). Gestión Organizacional. Chile: Universidad Católica de Chile.

9. Robbins, S.P. (2004) Comportamiento organizacional. México: D.F. Prentice-Hall Hispanoamericana, S.A.,

10.Sandoval; P. (2004). La capacitación en la empresa. Barcelona: Editorial Sirio, S.A

\section{REFERENCES CONSULTED}

1. Aldana, J., \& Piña, J. (2017). Quality of service provided to the client by gym instructors. Interdisciplinary Arbitrated Review Koinonía, 2 (3), 172-197. Recovered from http://fundacionkoinonia.com.ve/ojs/index.php/revistakoinonia/article/view/59/46

2. Brunet, L. (2007). Climate in Organizations. Panapo editorial. Mexico.

3. Cavassa, C. (2005). Industrial Safety an Integral Approach. Mexico: Limusa.

4. Gotera, F. (2005). Communication in organizations. Panapus editorial. Buenos Aires. Argentina.

5. Goncalves (2002). Fundamentals of Organizational Climate. Latin American Society for Quality. (SLC).

6. Hernández, Fernández and Baptista (2014). Investigation methodology. Mexico, Mc Graw Hill Hispanic American. Hill International 


\section{CIENCIAMATRIA}

Revista Interdisciplinaria de Humanidades, Educación, Ciencia y Tecnología

Año V. Vol. V. N9. Julio - Diciembre 2019

Hecho el depósito de ley: pp201602FA4721

ISSN-L: 2542-3029; ISSN: 2610-802X

Universidad Nacional Experimental Francisco de Miranda (UNEFM). Santa Ana de Coro. Venezuela

Carlos Enrique Santis Loor; Alba Verónica Barreto Pincay; Flor Maria Bailón López; Maria Monserrate Bravo Guerrero

7. Iglesias, F. (2008), Observation and Evaluation of the Learning Environment in Early Childhood Education: Dimensions and Variables to Consider. Iberoamerican Journal of Education. Publication edited by OEI № 47

8. Ramírez, F. (2005). Organizational Management Chile: Catholic University of Chile.

9. Robbins, S.P. (2004) Organizational behavior. Mexico DF. Prentice-Hall Hispanoamericana, S.A.,

10. Sandoval; P. (2004). The training in the company. Barcelona: Editorial Sirio, S.A

(C2019 por los autores. Este artículo es de acceso abierto y distribuido según los términos y condiciones de la licencia Creative Commons Atribución-NoComercial-Compartirlgual 4.0 Internacional (CC BY-NC-

SA 4.0) (https://creativecommons.org/licenses/by-nc-sa/4.0/). 